\title{
Determination of the parameters of a vortex ring with an air core in a liquid by computer processing of video images
}

\author{
E.A. Chashnikov ${ }^{1}$, V.V. Nikulin² \\ Lavrentyev Institute of Hydrodynamics SB RAS \\ 1 ORCID: 0000-0002-1775-1703, chashnikov.e.a@gmail.com \\ 2 ORCID: 0000-0001-8996-6490, nikulin@hydro.nsc.ru
}

\begin{abstract}
The aim of this work is to study vortex rings with high buoyancy. Due to the vertical upward injection of a pulsed air jet into water, an air ring is formed, the shape of which is close to a torus, but not stationary in time and inhomogeneous in space. To compare experiments with theory, it is necessary to know the average geometric sizes of the ring. An autonomous method for processing vortex ring's video images obtained from two mutually perpendicular directions: from the side and from above, has been developed in the Matlab. The proposed technique can be ported to other programming languages and allows to measure dependences of the distance travelled, the core mean radius, the core cross-section mean radius and core volume on time by defining the visual boundaries of the ring from both directions. The technique does not depend on the background uniformity. Necessary condition is a leap in the intensity of the black-and-white image between the background and the entire ring boundary. Visualization was carried out by the shadow image method. The threshold contrast leap in our case was equal to 4 . Processing of a colour image is possible after conversion to black and white.
\end{abstract}

Keywords: vortex ring, buoyancy, toroidal bubble, image processing, scientific method.

\section{Introduction}

This work is devoted to the method for processing shadow video images of vortex rings with a gas (air) core in water, and its experimental approbation. A vortex ring with a gas core in a liquid is also called a toroidal bubble $[1,2]$, since the gas volume has a shape close to a torus. Such a vortex is a particular case of a wider class of floating vortex rings. Floating vortex rings differ from vortex rings in a homogeneous liquid in that they contain a fluid within themselves, the density of which is less than the surrounding one. As a result, a buoyant force acts on them, directed against the acceleration of gravity. The relevance of the study of floating vortices is due to their frequent occurrence in technogenic and natural processes. They can be formed, for example, during explosions, pulsed emissions of liquid or gaseous fluid with a density different from the environment during man-made processes or accidents, volcanic eruptions, thermals formation, by dolphins [3 - 6]. Note that the vortices generated by the explosion have found practical application for extinguishing fires in oil and gas wells [7]. Recently, the urgency of studying the dynamics of toroidal gas bubbles in a liquid has grown in connection with the creation of screens that damp low-frequency acoustic noise when using them [8]. This is due to the fact that only toroidal bubbles remain stable at sufficiently large sizes required for this purpose. Such screens can be used to protect marine mammals from noise arising from the installation and operation of wind power generators on the offshore. In addition, the study of vortex rings with a gas core 
in a liquid is of broader importance, since their example may help to identify various regularities in the dynamics of floating vortex rings as a whole.

In the experimental study of vortices with a gas core, it is necessary to be able to determine in the process of motion such parameters as the volume of the gas, the characteristic dimensions of the toroidal bubble, and the speed of motion. This information is required for comparison with the available theoretical models of floating vortex rings $[9,10]$. Graphic methods for processing experimental images previously used in the field of studying vortex rings had a rather limited functionality that did not correspond to actual needs. For example, in $[11,12]$, the parameters of vortex rings were measured manually using shadow video images. In [13], software processing was used to measure the vortex shadow image darkening in the mass transfer process and some of its parameters, the exact shape of the core not being determined.

In the experiments carried out, the vortex rings have large air cores of complex shapes; therefore, direct measurement of their parameters by manual methods is impossible due to the need to measure the dimensions of the core in many sections and to average them. It should be noted that such inhomogeneity is a typical phenomenon for experiments with large air cores. In the photograph presented in $[14,15]$, the shape of the bubble core is also inhomogeneous in space. However, the method for measuring the vortex parameters is not described there. So, the determination of the geometric dimensions of the specified air ring is an urgent task. In this way, the task of developing a software method was posed and solved. This software allows us to determine the evolution of the vortex ring air core parameters as it moves and to compare the experimental results with the previously developed theoretical model, which will enable the model to be tested under fundamentally new conditions. Dependences of the distance travelled, the core mean radius, the core cross-section mean radius on time, and its initial volume are required to compare the experimental data with the conclusions of the analytical model [10].

\section{Experimental setup}

The experimental setup is transparent vertical plexiglass vessel with water with a cross section of $195 \times 220 \mathrm{~mm}^{2}$ and a height of $1200 \mathrm{~mm}$. At the vessel base, at a distance of $100 \mathrm{~mm}$ from the bottom, there is a nozzle with a diameter of $4 \mathrm{~mm}$. Air is supplied from the compressor at a pressure of 2 to 6 atmospheres for short periods of time from 10 to $40 \mathrm{~ms}$. Due to the vertical upward injection of a pulsed air jet into water, a vortex ring with air core is formed, the core shape is close to a torus, but not stationary in time and inhomogeneous in space. Air trapped in the vortex core is contained inside the core throughout the entire path of the vortex ring.

A mirror is installed in the upper part of the vessel at an angle of 45 degrees, which allows to simultaneously register the vortex ring both from the side, perpendicular to the direction of movement (vertical projection) and from above, along the direction of movement (horizontal projection). To use the developed image processing method, which will be presented below, no special lighting conditions are required, for example, background uniformity. The only and important condition is the sufficient amount of contrast between the vortex ring and the background. Registration was carried out by the method of shadow image on a light background. An LED lamp was used as the background for the side view, and for the top view, a white matte plate located under the nozzle in the vessel and illuminated by two halogen lamps.

Registration was carried out on a high-speed MotionalXtraHG-10ok camera with a frame rate of 500 fps and an exposure of $125 \mu \mathrm{s}$. The camera was located at a distance of $6200 \mathrm{~mm}$ from the vessel, that allows eliminating 
the change in scale when registering the vortex motion from the side and to minimize the change in scale when registering from above. The characteristic vortex diameter was 50-70 $\mathrm{mm}$, and the path was $300 \mathrm{~mm}$. The camera shoots in black and white. The proposed method for measuring the ring parameters is applicable to a colour image. Image processing was implemented in the Matlab environment using the Image Proccesing ToolBox and basic elements. In particular, new functions in the field of vortex ring image processing, functions edge, grayconnected, bwconcomp, bwboundaries, regionprops, rangefilt, imadjust were used.

\section{Vertical projection}

Side view registration is used to measure the law of vortex motion, that is, the dependence of the distance travelled on time. The rest of the parameters from this angle cannot be measured due to the core shape complexity and the ring plane deviation from the horizontal. The arithmetic mean of the boundary coordinates is taken as the coordinate of the vortex in space in the motion direction. The coordinate relative to the vortex obtained is shown in figure 1(d) with a white line. Accordingly, to determine the vortex position the knowledge of a bubble boundary is required.

The algorithm for determining the bubble boundary is similar for all images. Preliminarily, a fragment is cropped from the general image by imcrop, which includes only the area of motion of the vortex ring, but excludes all external hard boundaries, changes in colour intensity in the image, for example, a dark vessel and a nozzle against a light background.

In the first step, the edge function applied to the above image finds all contours with a sharp drop in intensity. The parameters in the function is the Canny method with a threshold equal to 10 and a standard deviation of the sigma filter equal to 0.9. The edge parameters were obtained empirically in such a way that only the boundary encircling the bubble was determined (figure 1(a)), and the background inhomogeneity was not taken into account.

In the experiments processed the ring was often followed by air bubbles of different sizes, but certainly smaller than those of the ring. In the vertical projection on the images they are always separate. The function edge returns a binary image that will include both the border of the ring and the border of the bubbles. The function bwconcomp, which finds and structures all connected, contiguous pixels with a value of 1 , are used to find all contours in a binary image. By this function it is easy to find the largest contour in terms of the number of pixels and remove all others by changing those value to 0 . The resulting contour lies close enough to the real boundary of the ring in order to calculate all the necessary parameters with high accuracy. However, figure 1(a) shows the ideal case, in some of the images the resulting contour has discontinuities, that is, it is not closed, and any algorithm for artificially completing the contour contradicts the idea of accurately identifying the entire border. The resulting contour allows the preliminary position of the vortex on the image to be automatically determined as the extreme vertical and horizontal contour points, which, due to shadow imaging, are always strongly darkened compared to the light background. Then a small rectangular fragment that contains a vortex ring is cropped along these points on a large image with indents of several pixels (figure 1 (b)). 

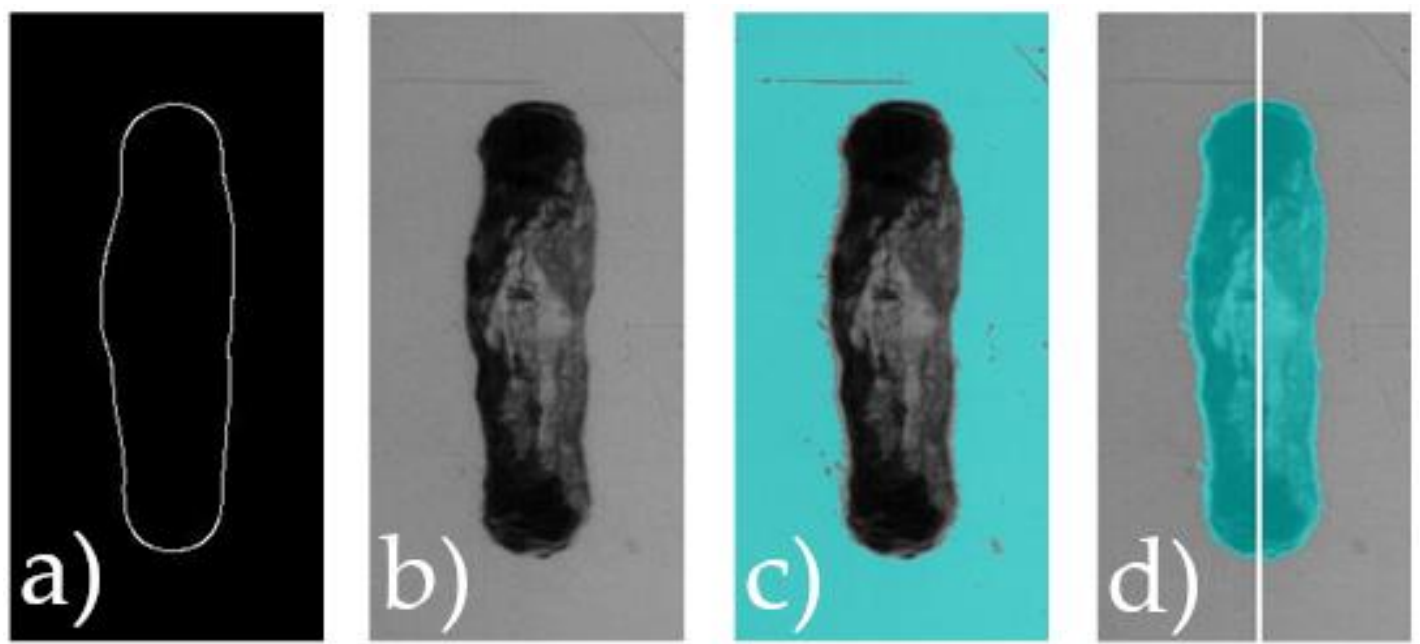

Figure 1. Typical vertical projection processing: (a) fragment with ring boundary from edge, (b) cropped from original image, (c) background selection, (d) typical coordinate and resulting selection.

At the second stage, the grayconnected function is used, which works on the magicwand principle by analogy with well-known graphic editors like PhotoShop. This function selects an area lying nearby and closes in intensity to the selected point and returns the matrix. The matrix size is equal to the image size, and nonzero matrix values correspond to the selected area. Applying this function to some points on the image border like figure 1(b) and adding the matrices, the region around the vortex ring is selected (figure 1(c)). The numerical scalar that determines the threshold difference of tolerance contrasts, in our case, is 4 . Such a small value of the threshold difference relative to the entire intensity scale of a black-and-white image of 256 units is enough to select the entire background, except for small irregularities, but at the same time not to select the ring. After binarization и inverting the selection (matrix), the bwconncomp or regionprops functions may be used to structure all connected pixels and calculate some properties, for example, the square of the resulting connected areas. Thus, by selecting the largest area and removing all the rest only the ring remains. The result of using this algorithm is shown in figure $1(\mathrm{~d})$. The obtained selection is seen to visually coincide with a high accuracy with the vortex ring. The vortex position is then calculated as stated above.

\section{Horizontal projection}

In case of horizontal projection, functions grayconnected, bwconncomp, bwboundaries, regionprops, rangefilt, imadjust are required. In this case, the vortex boundary extraction method is similar to the second stage of vertical projection image processing. Initially, a fragment containing only a vortex ring and a background is cropped (figure 2(a)).

Selection the external background with the grayconnected function along the border of the image either does not capture the shadow from the nozzle, or captures the ring if the tolerance parameter is too high. Nozzle shadows and other irregularities can be filtered with rangefilt, further increasing the contrast of the filtered image with imadjust. The rangefilt function replaces the intensity of each pixel in an image with the difference between the maximum and minimum intensities of neighboring pixels, thereby increasing the image contrast and darkening areas where the intensity changes smoothly. The use of grayconnected at the border of the filtered image allows one to select the vortex ring without significant deviations (figure 2(b)). 


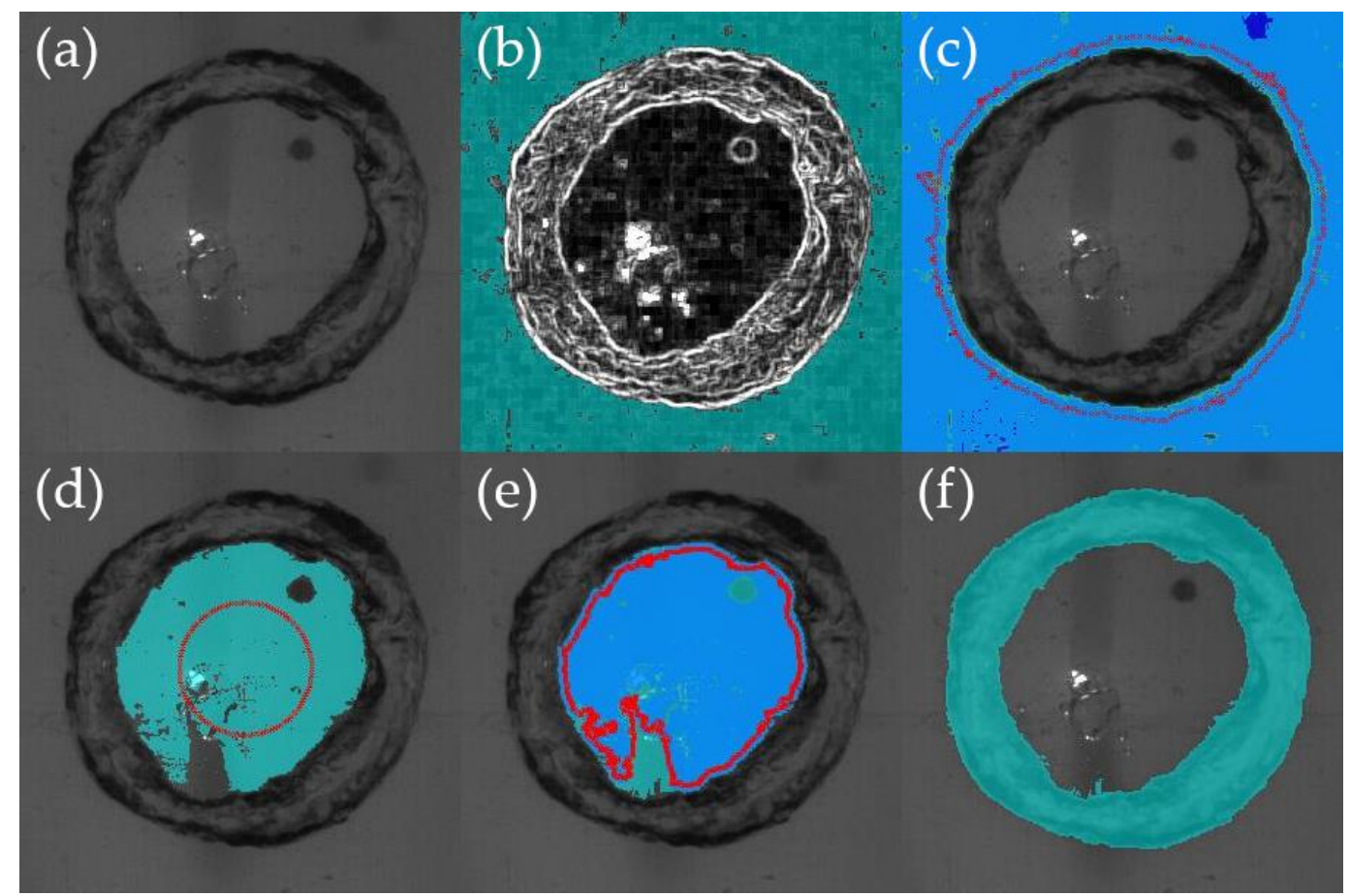

Figure 2. Typical horizontal projection processing: (a) original, (b) selection after filtering outside, (c) second selection outside, (d) first selection inside, (e) second selection inside, (f) resulting vortex selection. Green - selection area at the corresponding stage. Blue is the area of the previous selection. Red - coordinates of the grayconnected function application.

To increase the selection accuracy of the outer and inner borders, grayconnected function is applied closer to the ring border. To do this, after a rough selection of the external background, areas and boundaries of a small square and size are similarly removed. The selection is inverted. Next, the regionprops function is used to the selected area with the Centroid parameter, which returns the arithmetic mean of the coordinates of all points in the area, that is, the vortex conditional center. By the bwboundaries function one can return the coordinates of the border of this area. Thus, by shifting the resulting border a few pixels from the vortex center (red dots in figure 2(c)), allows applying grayconnected close enough to the ring in the original image without touching it. After re-selection of the outer border, the error becomes minimal (green + blue area in figure 2(c)).

For a rough selection of the inner area we apply a grayconnected function to points lying on a circle with a radius several times smaller than the radius of the outer border and center in the center of the vortex (red points in figure 2(d)). The imfill function fills in the empty areas of the resulting selection. Then, on the contrary, by a few pixels, pulling the border of the previous selection closer to the vortex center (red points in figure 2(e)), grayconnected selects the vortex inner region (figure 2(e), green area + blue area). The result of the inverted alignment of the inner and outer regions is the core, which shown in figure 2(f).

Forward angular integration is not suitable for obtaining the average vortex parameters, since it depends on the conditional center of the vortex, which essentially depends on the vortex. To measure the vortex parameters, a calculation by the method of equivalent diameters calculated by the regionprops function with the EquivDiameter parameter was chosen. The method of equivalent diameters is to replace the selection with a circle of equal area. That is, the core radius and the core cross-section radius were 
calculated as the half-sum and half-difference of the equivalent circle's radii of the outer and inner torus boundaries. The vortex ring volume was calculated as the volume of the torus $V=\frac{4 \pi}{3} \cdot R a^{2}$, where $R$ is the core radius, $a$ is the core cross-section radius, $V$ is the core volume.

a)

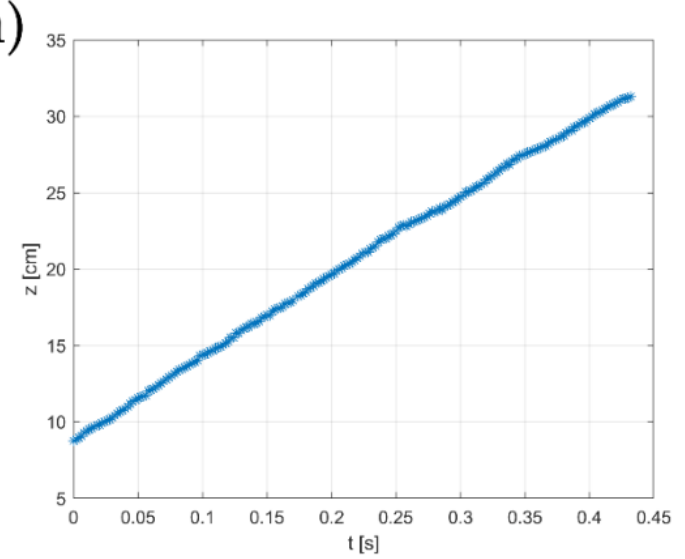

c)

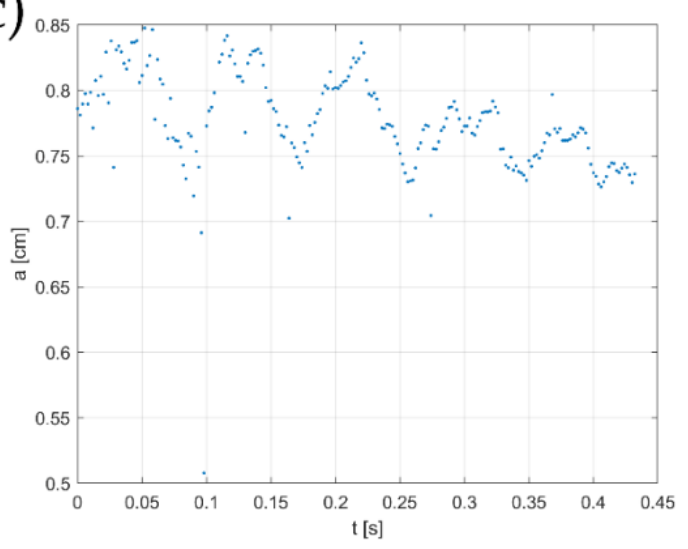

b)

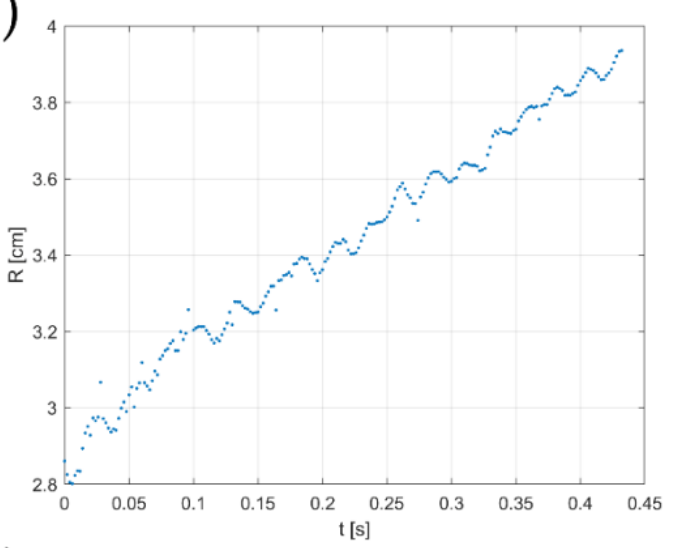

d)

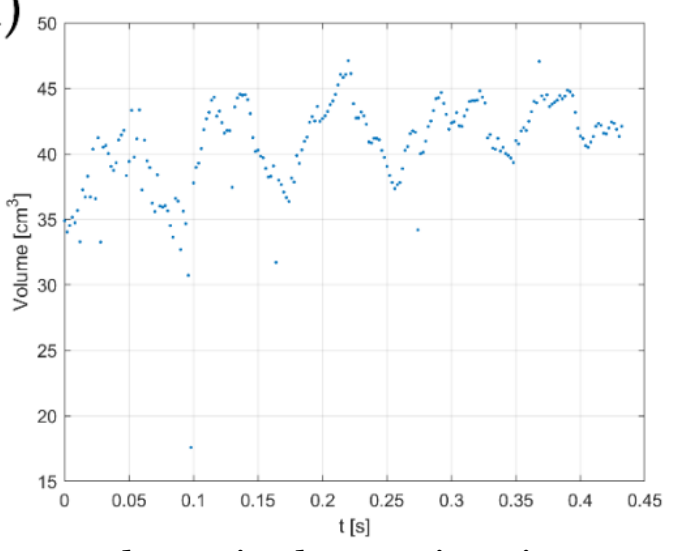

Figure 3. The resulting graphs for the experiment shown in the previous images: dependences (a) travelled path, (b) radius, (c) radius of cross section, (d) volume of the core on time.

The obtained parameters from the horizontal projection are multiplied by the perspective coefficient, that is calculated from considerations of geometric optics as follows: $r=r^{\prime}\left(1+\frac{l}{L}\right)$, where $r$ is the real size, $r^{\prime}$ is the size in the mirror, $l$ is the distance from the mirror to the vortex, $L$ is the distance from the camera to the mirror. In the experiment, $l$ varied from 315 to $15 \mathrm{~mm}$.

\section{Results and discussion}

The developed method allows automatic registering of all necessary vortex ring parameters with high accuracy for comparison with the analytical model [1]. The law of motion is constructed, in which the nozzle position is taken as the zero traversed path of the vortex, $\mathrm{z}=\mathrm{o}$. The intersection of the vortex with a certain coordinate 8 centimeters from the nozzle, where it can be considered formed [4], is taken for the zero moment of time. The dependencies of the radius of the core, the radius of the cross-section of the core, and the volume on time are also constructed (figure 3). Based on similar results, the experimental results will be compared with the conclusions of the analytical model.

The advantages of the proposed method may include its complete autonomy, which allows processing of large series of images of the same type without user participation, 
high accuracy in measuring the required parameters, and the ability to analyze the shape core dependency on time. Method implementation relies only on the basic Image Toolbox, all stages of video image processing can be ported to other programming languages.

The proposed method has several disadvantages. In a number of images, some areas at the vortex ring border are equal in intensity to the background, that is, they are visually indistinguishable, which leads to the selection of a ring part along with the background and, accordingly, incorrect results. Points that significantly fall out of the general dependence in figure 3 correspond to those images. Touching the bubbles border following the vortex with the inner border of the vortex leads to an accidental error at the initial stages of the vortex movement, since it is almost impossible to fully separate them, and the selection of a bubble can lead to a vortex selection. These errors can be taken into account visually based on the obtained dependencies, or by creating an automatic differential filter, which being based on the absolute difference in values between adjacent points can sweep out points with a difference exceeding a certain limit value.

Currently, a search is underway for other areas where the proposed methodology may be useful. The possibility of using machine learning to reduce the contrast requirements between the ring and the background is also being considered.

\section{References}

1. Walters J. K., Davidson J. F. The initial motion of a gas bubble formed in an inviscid liquid // Journal of Fluid Mechanics, Vol. 17, 1963, pp. 321-336.

2. Lundgren T. S., Mansour, N. N. Vortex ring bubbles // Journal of Fluid Mechanics, Vol. 224, 1991, pp. 177-196.

3. Akhmetov D. G., Kotelnikova M. S., Nikulin V. V., Plastinin A.V., Chashnikov E.A., Kop'ev V. F., Zaitsev M. Y. Generation of Large-Scale High-Velocity Vortex Rings by Initiating an Explosive// Combustion, Explosion, and Shock Waves, Vol. 55, № 4, 2019, pp. 390-394. Doi: 10.1134/So010508219040038.

4. Maxworthy T. Some experimental studies of vortex rings// Journal of Fluid Mechanics, Vol. 81 № 3, 1977, pp. 465-495. doi:10.1017/Soo22112077002171

5. Lesage, P., Kemiha M., Poncin S., Midoux, N., Li H.Z. Mimicking Dolphins to Produce Ring Bubbles in Water// Biomimetics, Vol. 1, № 6.2016. https://doi.org/10.3390/biomimetics1010006

6. Zhou X., Xu Y., Zhang W. Formation regimes of vortex rings in thermals// Journal of Fluid Mechanics, Vol. 885, A44, 2020. doi:10.1017/jfm.2019.1036

7. Akhmetov D. G. "Vortex Rings." Springer, Berlin (2009).

8. Wochner M., Hinojosa K., Lee K., Argo T., Wilson P., Mercier R. Acoustic behavior of large encapsulated gas bubbles with resonance frequencies in the $50-100 \mathrm{~Hz}$ range // The Journal of the Acoustical Society of America, Vol. 127, 2015. Doi: 10.1121/1.3385244.

9. Turner J. S. Buoyant vortex rings // Proceedings of the Royal Society of London. Series A, Mathematical and Physical Sciences. Vol. 239, 1957, pp. 61-75.

10. Nikulin, V.A. Analytical model of motion of turbulent vortex rings in an incompressible fluid // Journal of Applied Mechanics and Technical Physics., Vol. 55, 2014, pp. 558-564. 10.1134/So021894414040026.

11. Nikulin V.V. Motion of a buoyant vortex ring opposite to the lift direction // Doklady Physics, Vol. 61, № 2, 2016, pp. 74-77

12. Nikulin V.V. Dynamics of vortex rings moving counter the lift // Fluid Dynamics, Vol. 52(1), 2017, pp. 88-93. 
13. Nikulin V. V., Panenko R. A. Experimental observation of turbulent exchange between heterogeneous vortex ring and surrounding medium by the shadowgraph method // Journal of Physics: Conference Series, 2019, pp. 012006. DOI 10.1088/17426596/1421/1/012006.

14. Vasel-Be-Hagh A. R., Carriveau R., Ting D. S.-K. A balloon bursting underwater // Journal of Fluid Mechanics, Vol. 769, 2015, pp. 522-540.

15. Hershberger R., Bolster D., Donnelly R. Slowing of vortex rings by development of Kelvin waves // Physical review. E, Statistical, nonlinear, and soft matter physics. Vol. 82, 2010, pp. 036309. DOI:10.1103/PhysRevE.82.036309. 